\title{
Delusions in the phenomenological perspective
}

\author{
Andrzej Kapusta \\ Department of Philosophy and Sociology \\ Marie Curie-Sklodowska University in Lublin \\ andrzej.kapusta[Ipoczta.umcs.lublin.pl \\ Received August 2014; accepted December 2014; published winter 2014/2015.
}

\begin{abstract}
The aim of the article is to present the contemporary concepts of delusions from the phenomenological perspective. The difficulties to define delusions and the examples of delusional disorders, such as delusional mood, Cotard's syndrome, or Capgras delusions, serve as the point of departure for this analysis. The questions of the phenomenological understanding of delusions are presented in the context of Karl Jaspers' theory of the incomprehensibility of psychotic thinking (primary delusions, delusional mood). The subsequent analysis presents the constraints of contemporary cognitive theories of delusions. The criticism of said theories highlights the need for creating a broader concept of experience and cognition and results in the proposition to use the terms "background" and "embodiment" as theoretical tools for a more complete understanding of the peculiarities of delusional experience.
\end{abstract}

Keywords: Delusional experience; Capgras and Cotard delusions; phenomenological psychiatry; Karl Jaspers; sense of reality.

\section{Phenomenological psychiatry}

The term „phenomenology” has been used in a variety of ways in phenomenological psychiatry with its specific meanings changing over time and with regard to particular contexts. The word was introduced to psychiatry by Karl Jaspers (Fuch and Stanghellini 2013) but while some psychiatrists, including E. Minkowski, E.W. Straus and V.E. von Gebsattel used it without a direct reference to philosophical tradition, others, e.g. L. Binswanger, tried to adopt the ideas of such thinkers as Husserl, Heidegger or Merleau-Ponty (Spiegelberg 1972). The basic goal of phenomenological psychiatry was not to explain the complex mechanisms behind delusional symptoms, but rather to provide an insight into the elements of psychotic experience. Gaining a first- 
hand account and considering the subjective processes that build up such subjective experiences, including perceptual meaning, temporality and smooth bodily actions, allows for the detection of mental distortions and mental disintegration. Phenomenological researchers explore not only the critical points in the constitution of human experience, but also the modes of striving for a coherent view, even if it is only possible in the form of delusions (Fuchs 2010: 548).

In contemporary psychiatry, the term "phenomenology" refers to the study of signs and symptoms as well as their underlying thoughts and emotions. Phenomenology acquired a meaning which differs from the one used by Jaspers and provides the basis for building the nosological system and classification. Nancy Andreasen (2007) expressed the view that such a classification system (DSM) leads to many inadvertent consequences. The descriptive criteria of diagnosis can only account for some of the important symptoms of a given disorder and are only the „gatekeepers” of a successful diagnosis. Such criteriology reduces the diagnostic system to a mere „check-list”, which has a dehumanizing impact on the psychiatric practice. Diagnostic criteria provide psychiatrists with a common nomenclature which offers reliability but lacks in validity (Kraus 1994).

Jaspersian phenomenology concentrated on a patient's "inner experience", with a particular significance attributed to spontaneous expressions and behaviours. Individual cases were interpreted as more informational than statistically generalised empirical data. In the light of the controversy related to the real nature of phenomenological psychopathology, let us consider its basic scope and aims (see Stanghellini 2011) ${ }^{10}$

1 Phenomenological psychopathology is not aimed at simply cataloguing mental symptoms. Its purpose is to comprehend patient experiences and understand the types of human existence.

2. Phenomenology seeks to understand the disorders affecting an individual by grasping the fundamental and elusive changes in the way patients experience reality and their own agency. Therefore, there is no need to focus only on florid symptoms such as delusions or hallucinations.

3. Psychotic persons experience a specific existential modification characterised by depersonalization, derealization and de-socialization. The analysis should concentrate on changes including self-perception, embodiment, sense of reality and communication with others.

\footnotetext{
${ }^{10}$ Critical statements about certain ways of practicing phenomenology are repeatedly discussed in the philosophy of psychiatry. Stanghellini (2004) and Matthews (2007) want to defend phenomenological analysis of the disorder against the "defect" of solipsism. They differentiate Husserlian egological phenomenology (with transcendental reduction) from his (or parallel) later statements on the lifeworld (see. Parnas et al. 2013, Naudin 1999).
} 
4. Analyses should be sensitive to the intersubjective and social contexts. Patients' perception of the world is sometimes radically different from most people's. Schizophrenic depersonalization consists in an impairment of common sense and disembodiment-the feeling of emotional distance and personal detachment.

5. This particular experience is not always directly reflected on and referred by patients. Its effects are manifested in their perception of the world and their social behaviour.

Phenomenological psychopathology reveals the "deeper" phenomena, as opposed to the "surface symptoms" of contemporary classification systems (DSM). A pathological syndrome -should be grasped within the profound transformation of subjectivity in its entirety. Syndromes are understood as derailments and distortions of the multitude of synthetic processes necessary for the constitution of human experience. As observed by Stanghellini, "Phenomenological thinking argues for a "third way," in between "surface" assessment as performed by descriptive and clinical psychopathologies, and the kind of "profundity" explored by "depth" psychologies" (Stanghellini 2011: 161$)^{11}$.

Before proceeding to a phenomenological analysis of delusions, let us first concentrate on the problems with their definition.

\section{Defining delusions}

Delusions are easy to diagnose but hard to define. In the classic definition, Jaspers describes delusions as false judgement held with strong certainty, impervious to contrary experience or argument and with impossible content (Jaspers 1963). The American Psychiatric Association defines delusions in a similar way. According to DSM-IV-TR, delusion is "a false belief based on incorrect inference about external reality that is firmly sustained despite what almost everybody else believes and despite what constitutes incontrovertible and obvious proof or evidence to the contrary...” (DSM 2000).The problem of the classic definition is that, although it offers an apt description of most delusions, it is thought to be unable to capture their salient features. Furthermore, the definition seems to be too broad in some cases, and two narrow in others. If the definition is too broad, it may appear to include the phenomena which are not delusions. This has serious practical consequences, as delusions, being a significant criterion for schizophrenia, constitute a legitimate basis for involuntary commitment. Sometimes, beliefs untypical of a given culture may appear incomprehensible given the patient's background or education, but

${ }^{11}$ Phenomenology employs rich philosophical terminology, a methodology in the form of "reduction" and the "principle of all principles", which researchers try to refer, with varying degrees of success, to psychopathological phenomena (Naudin 1999, Stanghellini 2005, 2011). 
are not necessarily pathological. Thus, it is easy to regard any novel, or even brilliant ideas as pathological, regardless of whether they subsequently turn out to be true or false. Furthermore, deep belief impervious to external argumentation need not always be regarded as a pathological symptom. The last classification system DSM-5 proposes the following formula: "Delusions are fixed beliefs that are not amenable to change in light of conflicting evidence. Their content may include a variety of themes (e.g. persecutory, referential, somatic, religious, grandiose).[...] Delusions are deemed bizarre if they are clearly implausible and not understandable to same-culture peers and do not derive from ordinary life experiences. [...] The distinction between a delusion and a strongly held idea is sometimes difficult to make and depends in part on the degree of conviction with which the belief is held despite clear or reasonable contradictory evidence regarding its veracity." The changes in the up-to date definition are not significant but some earlier theoretical arguments and critical discussions were taken into consideration (Heckers et al. 2013).

Attempts at regarding delusions as beliefs are frequently questioned. This is particularly the case if we define a belief as a statement which has been accepted on the basis of facts, evidence, or authority. In practice, judgement, perception, thought, sensation, or mood may also be delusional (Fuchs 2005). Supposedly, delusions may constitute only one element of a patient's distorted world, a world that partly overlaps numerous beliefs accepted in a given culture. For this reason, it is hard to separate certain delusions from false claims about reality, judgement, or recollection of previous experiences.

Gipps and Fulford (2004) suggest that the official definitions of delusions are able to capture the main criteria for the classification of delusions, though they do not identify their most salient features. The definitions focus solely on the most prominent external manifestations of delusions. As symptoms of schizophrenia ${ }^{12}$, such manifestations are the easiest ones to diagnose but also occur in other disorders. Thus, what we are left with are statistically determined indications of delusions. A deeper analysis would require discovering the ways in which delusions become bizarre, strange, and unfounded. Therefore, the focus should not be on the clear-cut form of delusions, but rather on the dynamics of their formation, especially their relation to other pathological phenomena, e.g personality disorders, and to the world experienced by the patient, especially the changes in bodily experiences, relations and ways of interacting with others. Only a proper conceptual framework will allow us to avoid fallacious and limited empirical hypotheses. ${ }^{13}$

\footnotetext{
${ }^{12}$ Delusions as symptoms are often present in patients with schizophrenia, but may also appear as symptoms in other mental and neurological cases. My main thesis is that delusional symptoms cannot be analysed as totally independent structure in human experience.

${ }^{13}$ From the phenomenological perspective we prefer a holistic analysis of human experience. We do not deny the value of experimental and narrow neuro-cognitive models and hypotheses (see interview with Jacob Hohwy in this volume).
} 
Phenomenological analysis seeks to determine the constituents of the natural attitude, in particular the attitude identified as a sense of reality. A deeper study of the structure of experience can shed some light on the experience of schizophrenic delusions. According to Stanghellini, the crucial part of schizophrenic experience is a crisis of common sense-the practical and habitual relation to the world. The phenomenological approach in psychiatry is not necessarily an implementation of its philosophical epoche (the theoretical moment of suspending all judgements about the existence of the external world, and consequently all action in the world), however, they can be recognized as a "shift from asking what is real and how we know what is real to asking what the sense of reality (an aspect of our experience that such questions take for granted) consists of" (Ratcliffe 2008: 9-10).

Delusions are often defined in epistemological terms as false and irrational beliefs which are the result of defective epistemic practices (Bortolotti 2008, Kapusta 2012). But delusions seen as beliefs do not always carry a pathological change in experience. Epistemic pathology need not imply existential pathology (Ratcliffe 2008: 282), as may be observed in terms of religious beliefs and prejudices. People with irrational and false beliefs can often function fairly well in everyday life. Phenomenological perspective, therefore, refers to the everyday, often pre-reflexive experiences of patients. As stated by Gipps and Fulford:

Delusions, far from being a product of everyday irrationality, thus seem to reflect a profound failure of that contact with reality which is presupposed by everyday reasoning from premises to conclusions. This is born out too by the point made in section I above, that it is not merely belief that can be delusional but also thought, evaluation, feeling, inner experience, empathic relating, perception etc. (Gipps, Fulford 2004: 229).

Delusions are basically a failure in the understanding of the world and other people. This essential non-ineligibility results from a change of the primordial engagement in the world, the lack of spontaneous interaction not mediated by any beliefs and representations (Kapusta 2010). The validity of this type of explanation may be evidenced by the presence of delusions, which are not only manifested through false, improbable and immodifiable beliefs, but also by perception, mood or inner experience. Analyses of the formation and persistence of delusions have shown how our thinking is limited by the number of prereflective, non-codified, background structures associated with corporeality (embodiment) and broadly understood affection (Rhodes, Gipps 2008, Stanghellini 2008).

A particularly important contribution to the understanding the phenomenological perspective is the division into primary and secondary delusions introduced by Karl Jaspers as a consequence of his thesis on the incom- 
prehensibility of schizophrenia (Kapusta 2013). The former, also referred to as delusion-like ideas, are meaningfully formed on the basis of the preceding affects, sensational, embarrassing or guilt-inducing experiences, false perceptions, derealizations, etc. Primary delusions are "quite alien modes of experience", largely incomprehensible and irreducible to any other experience or beliefs (Jaspers GP: 98 / AP: 82). A patient experiences primal sensations, feelings, moods, a kind of awareness that evokes a sense of alienation and a sensation of something being out of place. Jaspers gives the example of a patient who, when experiencing first symptoms of the disease, exclaimed: "Something is going on; do tell me what on earth is going on" (Jaspers GP: 98 / AP: 82)]. In Jaspers' interpretation, this kind of delusional atmosphere induces a kind of incomprehensible, distrustful and uncanny tension. Everything around acquires a new meaning, the perception of the environment is changed. An ambiguous delusional atmosphere may eventually bear fruit of finding something objective, the formulation of a more or less true belief, which strengthens and has a calming effect on the patient. One may become convinced that he is being persecuted, has committed a crime, or been accused of one (like Joseph K. in Franz Kafka's Process); one could also confidently foresee the coming of the golden age, have a sense of self-consecration or a conviction in one's own holiness (Jaspers GP: 98 / AP: 82).

Incorrigibility and certainty as an essential feature of primary delusion is associated, according to Jaspers, with a change of the patient's world to such an extent that its correction could pose a threat and a challenge to his very existence and individual world view. This kind of experience is frequently accompanied by the fully preserved capacity for formal, logical thinking and reasoning $^{14}$. Primary delusions may be developed into a coherent delusional system. However, it is built on the basis of a primary, incomprehensible experience that a patient wishes to associate harmoniously with real perceptions and other beliefs.

\section{Examples of delusions and theoretical discourse}

The Capgras and Cotard delusions serve as examples of monothematic and limited symptoms (Ratcliffe 2008, Radden 2011). These constitute a particularly valuable object of study, as their content basically repeats itself in various patients. The content of the former is limited to the belief in the existence of an impostor, whereas the latter consists in the patients believing that they are dead, or at least non-existent or disembodied. The above claims are not usually subject to deeper analysis. The belief that a friend or relative has been replaced by an impostor is impervious to other people's arguments

\footnotetext{
${ }^{14}$ Considerations about the role of false reasoning in psychotic symptoms see in the article by R.A. Adams and others in this volume.
} 
and the patient's own reflections that such a situation is unlikely and unsubstantiated. At the same time, the patient, while holding the belief that their relatives have been replaced, does not feel the need to search for them or investigate what has happened to them or whether they are safe.

The lack of coherence in beliefs or between belief and action begs the question of whether we speak of beliefs in the first place. Sometimes, the patients' actions are based on bizarre beliefs. The things they claim to be true cannot be just dismissed as nonsensical, and the very force of the claims proves the truth of their utterances. The patient may be in mourning for his wife or attempt to kill her impostor. Stone and Young (1997) see this kind of belief as a result of a loss of affective familiarity (the responsibility for which lies with the face recognition affective pathway) when attempting to recognise a familiar face. The cognitive function of recognition (overt visual pathway) is preserved. Capgras delusions, when put in this framework, constitute the opposite of prosopagnosia (the sensation of strangers being familiar), where affective (covert) face recognition is preserved while visual recognition is impaired ${ }^{15}$.

More detailed research indicates that the case of Capgras delusions is in fact more complex and we should indeed speak of a group of experiences and beliefs whose content may even pertain to such things as pets, familiar objects, or places. Such symptoms are not always experienced in isolation and they occur in the course of schizophrenia.

Professions of the annihilation of self, or in some cases, of the entire world occur in Cotard delusions. Claims such as "I am dead" or "I don't exist" express the patient's deep belief in his or her disappearance form the world, albeit not in reference to strictly biological criteria. Those claims should not be regarded merely as a kind of intriguing metaphor. Just as with Capgras delusions, we speak of the lack of coherence, or even contradiction in beliefs, which puts into question the possibility of ascribing this kind of claim to any propositional content. Clinical studies indicate a strong correlation between Cotard delusions and major depression, though, in most depressive disorders, the patients may claim to be feeling "as if" they were dead, and not that they are dead (Ratcliffe 2008).

Delusions are often accompanied by the emergence and development of paranoid schizophrenia. Klaus Conrad (1958) distinguishes four stages in this process: (1) the initial phase (das Trema); (2) the apophantic phase, i.e. the appearance of delusions. Often accompanied by a delusional mood (Wahnstimmung) and a transformation of the structure of experience; (3) the apocalyptic phase denoting the type of disintegration; (4) and the consolidation phase in stabilizing the patient's condition. The delusions emerging in the

${ }^{15}$ For a more precise analysis see the article by G. Young in this volume. 
course of (paranoid) schizophrenia are accompanied by the formation of a new structure in the person's experience and perception of the world. The phenomenological approach focuses on the structure of patient's experience and looks for relationships between the symptom and said experience in its entirety. This does not, however, preclude the biological substrate of schizophrenic disorders. In the tradition of research on schizophrenia, researchers have often treated itas an "epigenetic developmental process" and emphasised the patient's premorbid and preclinical susceptibility which is hard to diagnose because of its complexity and the lack of any clear symptoms. Bovet and Parnas (1993) speak of "defective" and "autistic attunement" (Parnas Bovet 1991), somewhat resembling Bleuler's "schizoid", Minkowski's "loss of vital contact with reality", Binswanger's "inconsistency of natural experience", or Blankenburg's "global crisis of common sense". From the phenomenological perspective it is difficult to treat "autistic vulnerability" and similar terms as delusions because they are accompanied by a change in the inter-subjective space and attunement to the patient's world. As observed by Bovet and Parnas: "Phenomenology sees autism as a defective expressive-perceptual attunement between the subject and the outer world. The autistic defect is perceivable both in the cognitive and affective domains because the attunement to the world is affected at a very elementary, preconceptual level" (Bovet Parnas 1993: 583).

Delusions are a kind of a thought disorder. They are examples of deep irrationality. As such, they are used in numerous philosophical discussions on the status of beliefs and criteria of rationality (Kapusta 2012, Bortolotti 2010). The research on cognitive processes responsible for the formation of delusions serves the purpose of producing theories explaining the common forms of thought and cognition (see also the article by M Coltheart in this volume). The search focuses on the differences between irrationality of our common beliefs, frequently manifested as superstitions or self-deception, and the deep irrationality of delusions. Contemporary psychiatric definitions of delusions focus on their external features, whereas the internal mechanisms of their formation receive little attention. These descriptions usually refer to epistemic terms, such as belief, justification, authenticity, or rationality. Special attention is devoted to the following: the imperviousness of delusions to rational critical argumentation, the intensity of their recognition, and their incompatibility with the commonly accepted content.

An individual symptom, however, is not a stand-alone "thing-like entity", but rather reflects the whole patient experience. Symptoms have a prototypical character which is not merely a social construct but is reflected in the revised structure of a patient's experience (e.g., self-awareness). Bovet and Parnas (1993) emphasize the close relationship between the nature of delusions and a patient's distorted (self/ego) experience (Ich-Storungen). Disturbances of selfperception, which is often referred to as "depersonalization", "derealization" 
or "disturbed ego-boundaries", enable researchers to capture important aspects of delusions. Delusions of grandiosity and delusions of control not only carry a certain bizarre content, but affect the essential dimensions of a patient's world. As described by Blankenburg: "In both cases (delusion of omnipotence and delusion of control) the link between I and the environment seems qualitatively changed in a characteristic way. It is not the direction, that is, it is not the question of either I or the environment being experienced as the more powerful, nor is it the unilaterally concerned mineness of experiencing; it is rather the border between "mine" and "yours" (i.e., the outer world), and not the border only, but also the way in which it is overstepped, that is specific for schizophrenia (Blankenburg 1988, p. 187 translated by Bovet and Parnas, 1993). It should also be noted that the content of delusions alone is not enough to attest to their pathological nature. Bovet and Parnas refer to the theme-based classification proposed by the Polish psychiatrist Antoni Kępiński (ontological, eschatological and charismatic trend) (Kępinski 1978). It is not the content itself that gives them a pathological character, but the resulting distortion of intersubjective relations with others and an intangible shift in the dimension of one's relationship to the world.

\section{In search of the adequate framework for understanding delusions}

Monothematic Capgras or Cotard delusions seem to share a certain, specific content. However, a broader analysis reveals that many aspects of a patient's world view and self experience cannot be reduced to irrational and false beliefs (Gallagher 2009). Interestingly, this kind of thematic delusions shows a great similarity with delusional atmosphere and its elusive content. As described by Jaspers: "Patients feel uncanny and that there is something suspicious afoot. Everything gets a new meaning. The environment is somehow different-not to a gross degree -perception is unaltered in itself but there is some change which envelops everything with a subtle, pervasive and strangely uncertain light. A living-room which formerly was felt as neutral or friendly now becomes dominated by some indefinable atmosphere. Something seems in the air which the patient cannot account for, a distrustful, uncomfortable, uncanny tension invades him". (Jaspers 1963: 98)

Jaspers defines delusions (not just delusional atmosphere) in terms of the creation of a sense of reality. Like Gipps and Fulford (2004), he notes that "experience, within which there is a delusion, is the experience and thinking that something is real" (Jaspers, GP: 92 / AP: 79). The sense of reality is a primary phenomenon, which only in theory can be separated from the thoughts and ideas of reality. Delusional experience includes: 
1. changes in the experience of one's own corporeality

2. loss of a practical relation to the world, separation from the social world (a kind of alienation)

3. distorted experience of one's own capacity to act and influence potential relationships with others.

Total change in being in the world is manifested by the change in one's experience of the background and of belonging to the world. Gipps and Fulford (2004) define delusions in terms of commitment and the initial pre-conceptual understanding of reality. Ratcliffe (2008) refers to a change in existential orientation or existential feeling. Such explanations of the nature of delusions cannot be obtained on the biological level and within the framework of existing cognitive models which refer to anomalous experiences (Maher 1999) and errors in reasoning (Bortolotti 2008, Kapusta 2012). Mental disorders result primarily in the loss of the sense of reality and alter the plane of possible interactions with objects and potential relationships with other people. Delusional ideas make explicit the things that are, in common experience, implicit and very familiar at the same time. Reconstruction of a delusional experience implies the existence of non-propositional knowledge or a background structure. In delusional (psychotic) cases, the background structure is unable to impose proper constraints on our thoughts, desires, or the ability to act and be committed to the world in an original way. Therefore, we speak of a more fundamental disorder of experience, rather than the absence of proper reasoning, or the failure to form the experiential content. A detailed analysis of experience would require a broader description of the basic structure responsible for the subject's correct functioning. Such a structure is referred to as embodiment (Gallagher 2005, Stanghellini 2008). A living body perceives elements of the world as being part of an activity it performs. Disembodiment, on the other hand, bears the consequence of common sense disorder and the absence of practical relation to the world (Kapusta 2010).

The phenomenological approach stands against the reduction of a disease phenomena to mere biological mechanisms and definition of mental disorders in terms of natural kinds. But this begs the question of the extent to which this kind of perspective can be complemented or accompanied by a nonphenomenological approach. Jaspers himself saw his methodological pluralism as capable of accommodating a significant number of different research methods on the condition that one remains aware of their characteristics and limitations. Mishara and Fusar-Poli (2013) recognize in Kapur's concept of "aberrant salience" a possibility to explain the "delusional atmosphere" in terms of the dopamine hypothesis of schizophrenia. Dopamine (dopaminergic system) mediates recognition of the significance of environmental stimuli and attentive processes of filtration and information selection. 
Many researchers believe that it is possible to reconcile the perspective of phenomenological research and neurocognitive science. The phenomenological method can provide reliable and insightful descriptions of various aspects of the experience (including pathological). It makes it possible to create a broader explanans for the cognitive science and to contribute to a better definition of the examined objects. On the other hand, the task of empirical science is to create objective models of cognition, which would explain how it is possible to have various phenomenal sensations, self-experience and feeling of reality. Varela and Gallagher (2001) argue that the interaction between phenomenology and science (e.g., psychopathology) may contribute to their mutual reinterpretation.

The phenomenological analysis of consciousness, mind, identity, and intersubjectivity may contribute to the development of the knowledge on psychiatric disorders. And psychopathology, by dealing with empirical cases of certain disorders of thought or self-experience, can contribute to the philosophical insight. What we expect from philosophy is a coherent and orderly structure which will be able to capture the complexity of human experience and behaviour. Whereas psychiatrists, in their studies on delusions, disorders of identity, problems of mutual understanding and communication cannot rely on naive, naturally assumed concepts of rationality, mind or reality.

\section{Bibliography}

American Psychiatric Association. 2000. Diagnostic and Statistical Manual of Mental Disorders, 4th Edition, text revised, DSM-IV-TR, Washington: American Psychiatric Association.

Andreasen, N. C. 2007. DSM and the Death of Phenomenology in America: an Example of Unintended Consequences, Schizophrenia Bulletin, 33(1): 108-117.

Bortolotti, L. 2010. Delusions and Other Irrational Beliefs, Oxford: Oxford University Press.

Conrad, K. 1958. Die beginnende Schizophrenic Versuch einer Gestaltanalyse des Ylahns. Stuttgart, Germany: Thieme.

Fuchs, F., and Stanghellini, G. 2013. One Hundred Years of Karl Jaspers "General Psychopathology”. Oxford: Oxford University Press.

Fuchs, T. 2010. Phenomenology and Psychopathology. S. Gallagher D. Schmicking. Handbook of Phenomenology and Cognitive Science. Springer: Verlag: 547-573.

Fuchs, T. 2005. Delusional Mood and Delusional Perception. A Phenomenological Analysis. Psychopathology, 38: 133-139.

Gallagher, S. 2005. How the Body Shapes the Mind, Oxford: Oxford University Press. 
Gallagher, S. 2009. Delusional Realities. W: Broome M. R., Bortolotti L. eds. Psychiatry as Cognitive Neuroscience. Philosophical Perspectives. Oxford: Oxford University Press: 245-268.

Gipps, R. G. T., Fulford, K. W. M. 2004. Understanding the Clinical Concept of Delusion. From an Estranged to an Engaged Epistemology, International Review of Psychiatry, 3: 225-235.

Jaspers, K. 1963. General Psychopathology. transl. J. Hoenig, M. W. Hamilton, Chicago: University of Chicago Press.

Jaspers, K. 1996. Allgemeine Psychopathologie. eds. 8, Berlin-Heidelberg: Springer.

Kapusta, A. 2010. Madness and Method: The limit of understanding in Philosophy and Psychiatry, Lublin: UMCS, [in polish].

Kapusta, A. 2012. The rationality of madness: philosophical debate over the contemporary concepts of delusions. Yevtukh, V. Radzik, R., Kisla, G., ed. Etnosociological and epistemological discourse in scientific space. Kiev: 229-245.

Kapusta, A. 2013. "Theory of incomprehensibility": social and environmental determinants of mental disorders, Annals of Agricultural and Environmental Medicine Ann Agric Environ Med 20(4): 832-837.

Kępiński, A. 1978. Podstawowe zagadnienia współczesnej psychiatrii. Warszawa: Państwowy Zakład Wydawnictw Lekarskich.

Kraus, A. 1994. Phenomenological and Criteriological Diagnosis. Sadler J. S., Wiggins O. P., Schwartz M. A. ed., Philosophical Perspectives on Psychiatric Diagnostic Classification. Baltimore: Johns Hopkins University Press: 148-162.

Maher, B. A. 1999. Anomalous Experience in Everyday Life. Its Significance for Psychopathology. The Monist, 82(4): 547-570.

Mishara, A, Fusar-Poli, P. 2013. The phenomenology and neurobiology of delusion formation during psychosis onset: Jaspers, Truman symptoms, and aberrant salience. Schizophrenia Bulletin, 39(2): 278-286.

Naudin, J., et al. 1999. The Use of the Husserlian Reduction as a Method of Investigation in Psychiatry, Journal of Cognitive Sciences, 6 (2-3): 155-171.

Parnas J, Sass LA, Zahavi D. 2013 Rediscovering psychopathology: the epistemology and phenomenology of the psychiatric object. Schizophrenia Bulletin, 2: 270-277

Radden, J. 2011. On Delusion. Abingdon \& New York: Routledge.

Ratcliffe, M. J. 2008. Feelings of Being. Phenomenology, Psychiatry and the Sense of Reality. Oxford: Oxford University Press.

Rhodes, J., Gipps, R. 2008. Delusions, Certainty and the Background. Philosophy, Psychiatry \& Psychology. 15(4): 295-310.

Spiegelberg H. 1972. Phenomenology in Psychology and Psychiatry. A Historical Introduction, Evanston: Nortwestern University Press.

Stanghellini, G. 2004. Deanimated Bodies and Disembodied Spirits. Essays on the Psychopathology of Common Sense. Oxford: Oxford University Press. 
Stanghellini, G. 2008. Schizophrenic Delusions, Embodiment and the Background. Philosophy, Psychiatry \& Psychology, 15(4): 311-314.

Stanghellini, G. 2011. Phenomenological Psychopathology, Profundity, and Schizophrenia. Philosophy, Psychiatry, \& Psychology, 18(2): 163-166.

Stone, T., and Young, A.W. 1997. Delusions and brain injury: the philosophy and psychology of belief, Mind \& Language, 12: 327-364. 